\title{
EVALUACIÓN DE AEROENFRIADORES DE UNA PLANTA DE FRACCIONAMIENTO DE LÍQUIDOS DE GAS NATURAL POR MEDIO DE RADIOTRAZADORES
}

\author{
EVALUATION OF AIRCOOLERS OF A NATURAL GAS LIQUID \\ FRACTIONNING PLANT BY MEANS OF RADIOTRACERS
}

Carlos Sebastián Calvo ${ }^{1}$

RECEPCIÓN: 03 DE SETIEMBRE DEL 2019

ACEPTACIÓN: 27 DE SETIEMBRE DEL 2019

\section{RESUMEN}

Se realizó el servicio en una planta de fraccionamiento de líquidos del gas natural para determinar la dinámica del diésel en un aeroenfriador, con la técnica de radiotrazadores para la detección en línea. El radiotrazador utilizado fue el yodo 131 (I-131), con una actividad total de $10 \mathrm{mCi}$. Se realizó el arreglo experimental respectivo en las diferentes partes del sistema estudiado, a fin de facilitar la inyección del radiotrazador a las líneas de ingreso del diésel al aeroenfriador y las respectivas detecciones que fueron consideradas a priori, de acuerdo con lo establecido en el procedimiento presentado en la etapa de implementación del servicio.

El sistema de adquisición de datos, al igual que los detectores en sus respectivas posiciones, estuvieron sincronizados y registraron valores de fondo de radiación ambiental (línea de base) antes de proceder a realizar la inyección del radiotrazador. Se registraron conteos de radiación a intervalos de 0.5 segundos.

Como resultado del análisis de los datos de la experiencia, se observa la presencia de algunas anomalías de flujo dentro del aeroenfriador, con tiempos de tránsito variables en algunos tramos y flujos preferenciales en la zona superior e inferior del sistema.

Palabras clave: radiotrazador, aeroenfriador, actividad, tiempo de residencia

\begin{abstract}
The service was performed at a natural gas liquid fractionation plant to determine the dynamics of the diesel in an aircooler, using the radiotracer technique for on-line detection, using $10 \mathrm{mCi}$ total activity of lodine 131 (I-131), as radiotracer. The respective experimental arrangement was carried out in the different parts of the system, in order to facilitate the injection of the radiotracer at the diesel input lines to the aircooler, and the respective detection points considered at the setting out of the experiment procedure, presented in advance, at the implementation stage the service.

The data acquisition system, as well as the detectors in their respective positions, were synchronized for recording the values of environmental radiation background (baseline), before radiotracer injection. Radiation counts were recorded at intervals of 0.5 seconds.

As a result of the data analysis from the experiment, some flow anomalies inside the cooler were observed, with variable transit times in some sections and preferential flows at some upper and lower part of the system.
\end{abstract}

Keywords: radiotracer, aircooler, activity, residence time. 


\section{OBJETIVO DEL ESTUDIO}

Evaluar la dinámica del diésel en un aeroenfriador de una planta de fraccionamiento de líquidos de gas natural utilizando la técnica de radiotrazadores.

\section{MATERIALES, INSTRUMENTOS Y EQUIPOS UTILIZADOS}

- 01 sistema multisonda de adquisición de datos DAMRI, equipado con nueve sondas de centelleo, y una computadora de campo con software para adquisición y procesamiento de información de radiotrazadores y fuentes de radiación

- Contador de radiación equipado con sondas de centelleo de $\mathrm{NaI}(\mathrm{Tl})$ para mediciones puntuales que utilizan radiotrazadores

- 01 monitor de radiación marca RADIAGEM 2000

- Sonda de detección de contaminación

- Dosímetros personales de radiación

- Cables para sonda de 30 a 100 metros.

- Blindaje cilíndrico para el material radiactivo

- Contenedores de plomo para I-131

- Jeringas descartables

- Guantes descartables

- Indumentaria diversa de protección personal

El estudio se complementó con la utilización del software Latin 36 (Francia) para procesar las diferentes curvas de respuesta del radiotrazador inyectado al sistema y detectado en las nueve posiciones indicadas.

\section{SISTEMA EVALUADO}

Una vista en perspectiva del sistema evaluado que indica la posición de los detectores de radiación D1, D2, D3, D4, D5, D6, D7, D8 y D9 se muestra en la figura 1, en la que se aprecia la entrada al aeroenfriador a través de dos tuberías equidistantes del eje central, en cuya vertical se encuentra la tubería de acceso.

En la zona de ingreso al sistema, existe un distribuidor del flujo de entrada en donde se posicionaron dos detectores de radiación. El ingreso del diésel al sistema ocurre en el nivel superior, que llamaremos primer paso, y llega al extremo opuesto, donde se observa el posicionamiento de otros cinco detectores de radiación, sobre un distribuidor que llamaremos distribuidor terminal o, simplemente, terminal. En este, se registran los diferentes pasos del diésel por esa posición durante su recorrido en el sistema.

Se indican, además, los cuatro recorridos del fluido por los diferentes pasos de tuberías, desde su ingreso hasta la salida del aeroenfriador. La detección a la salida del sistema enfriador se realiza a través de otros dos detectores. 


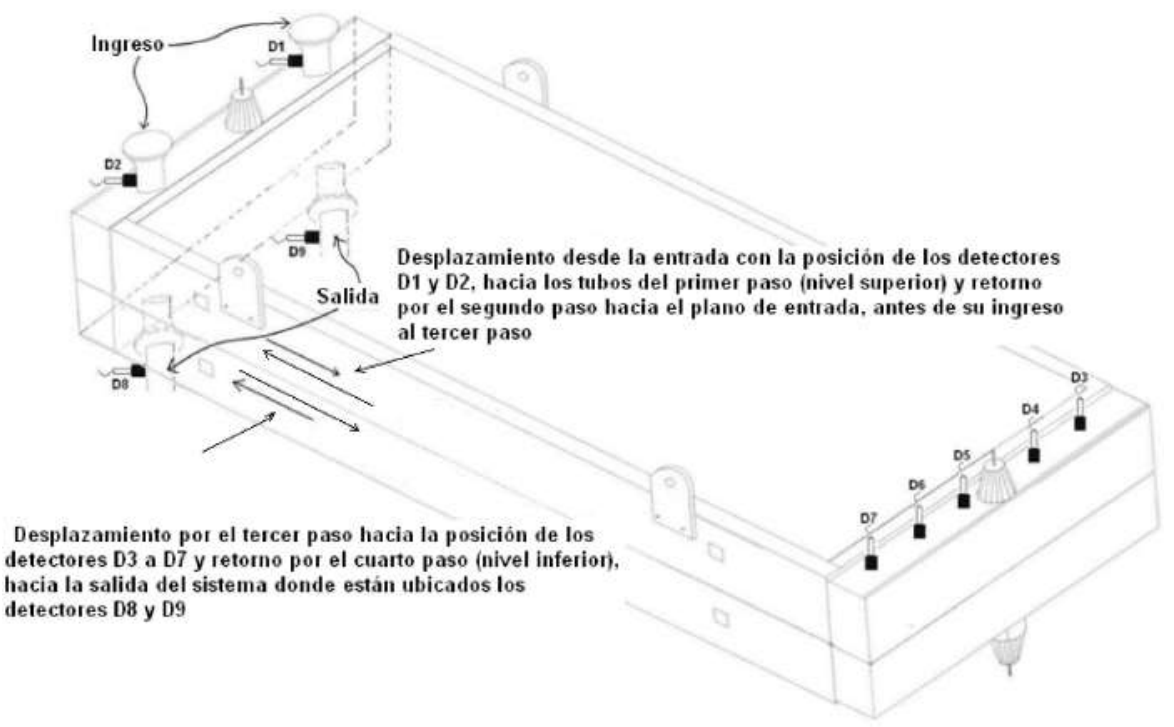

Figura 1. Vista isométrica del aeroenfriador evaluado. Elaboración propia.

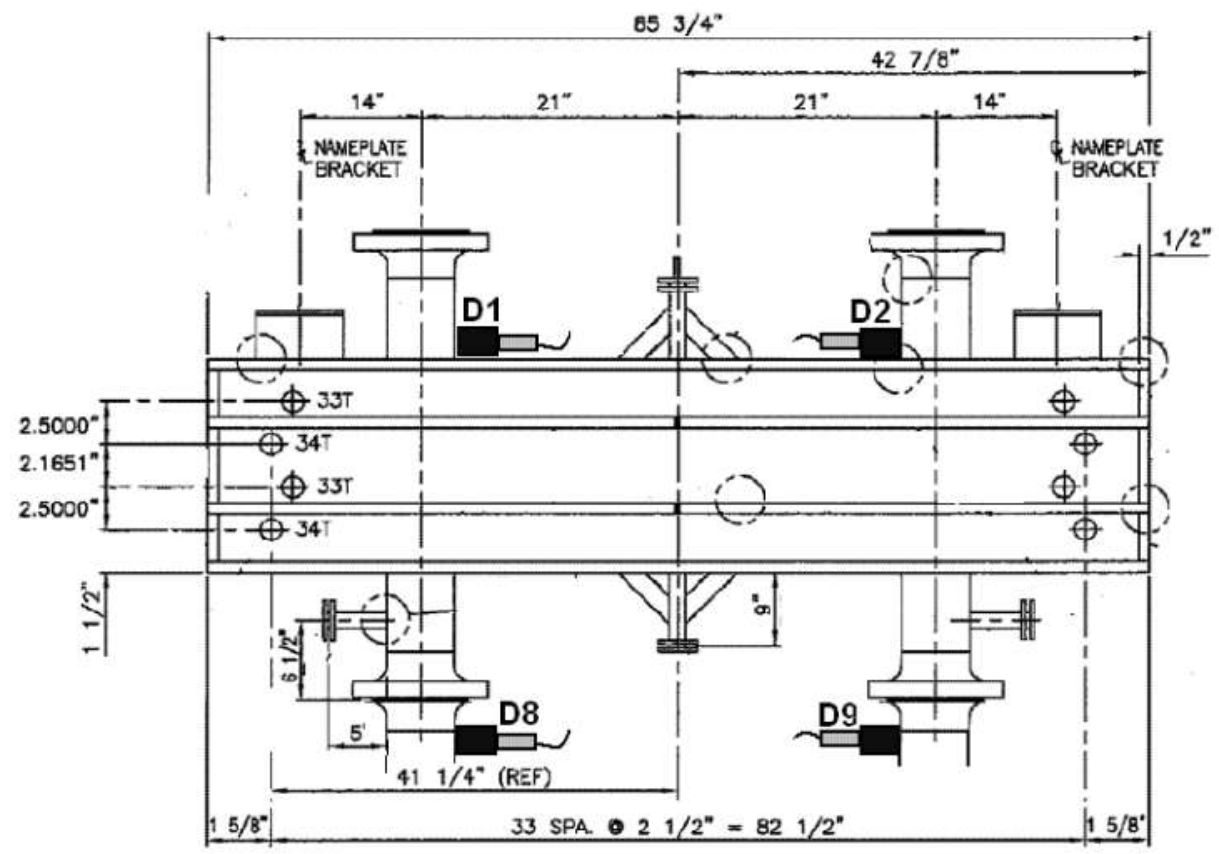

Figura 2. Vista frontal de la zona de entrada de diésel a un distribuidor ubicado en el nivel superior del aeroenfriador evaluado, justo antes del ingreso del fluido al primer paso del sistema. Se observan las dos entradas que se derivan de un solo ducto de acceso, con la instalación de los detectores D1 y D2 en cada una de ellas. Luego del recorrido por el sistema, se distinguen los ductos de salida del sistema por el cuarto paso o nivel inferior del aeroenfriador, en donde se han instalado los detectores D8 y D9. 


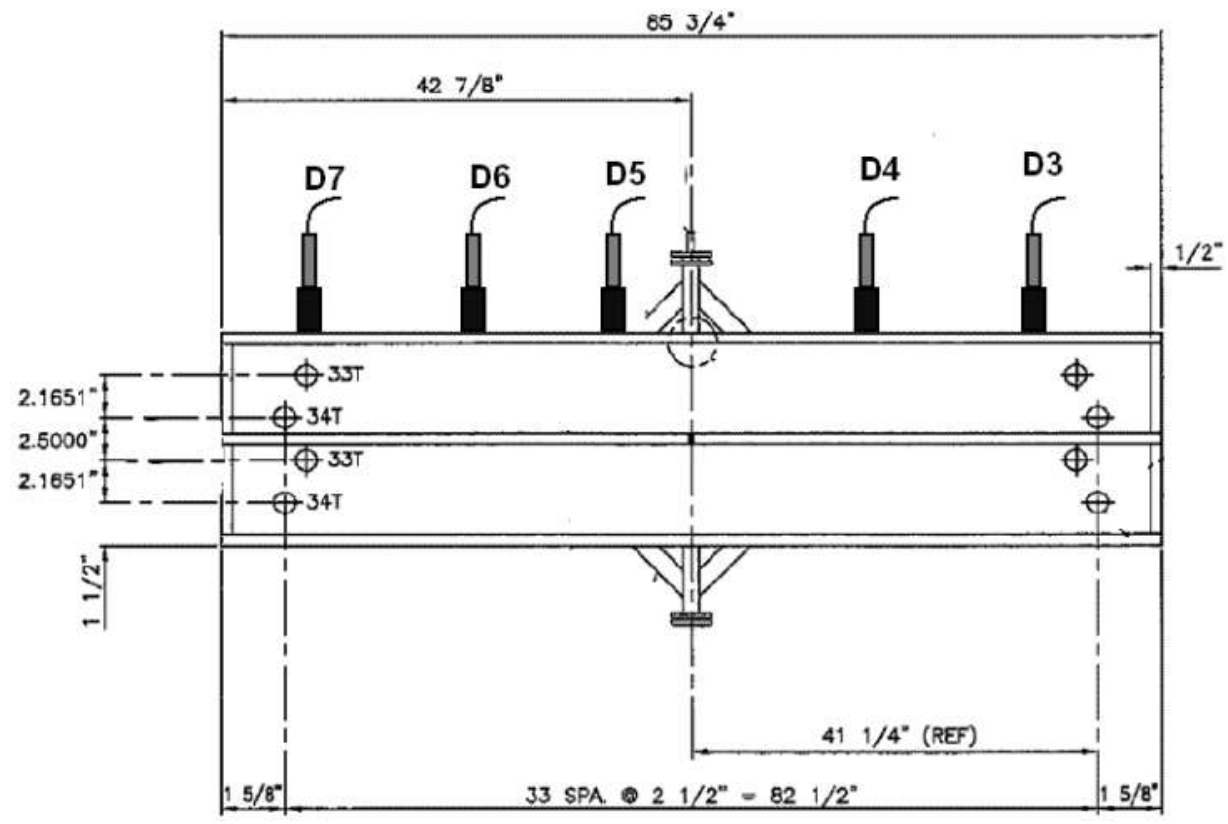

Figura 3. Vista posterior del primer recorrido de diésel por el primer paso o nivel superior del aeroenfriador evaluado para su ingreso a los tubos del segundo paso. Se observa la ubicación de los detectores D3, D4, D5, D6 Y D7 en el terminal posterior. Estos registrarán el final del recorrido por el tercer paso del aeroenfriador.

Como se observa en las figuras 2 y 3, el sistema cuenta en total con 134 tubos con aletas enfriadoras, representadas en la figura 4 . El sistema de enfriamiento consistente en un ventilador de álabes está ubicado en la parte inferior de la unidad evaluada.

$30^{\circ}-0^{* \prime}$

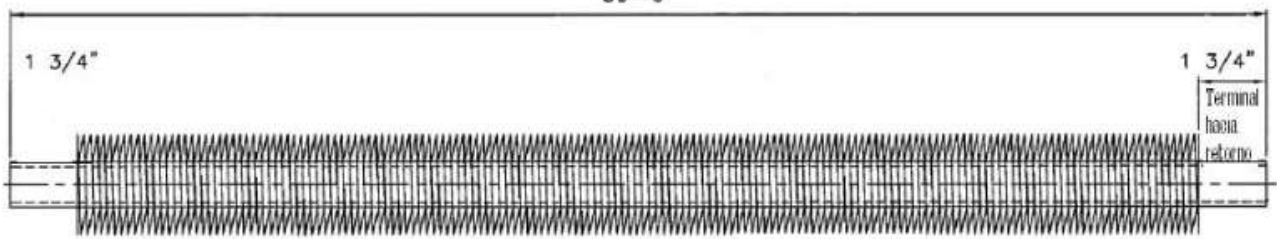

Figura 4. Vista de los tubos que recorren el enfriador, mostrando las aletas enfriadoras.

\section{Elaboración propia.}

Consideraciones teóricas a tener en cuenta en el sistema evaluado

El tiempo medio de tránsito $\bar{t}_{\text {exp }}$ se obtiene a partir de la curva de concentración del trazador a la $\bar{t}$ salida del proceso. En este caso, se obtiene lo siguiente:

$$
\bar{t} \exp =\int_{0}^{\infty} t C(t) d t
$$


Se sabe, también, que el tiempo de tránsito promedio geométrico se puede determinar considerando que la densidad del material permanece constante. Es una alternativa posible tomar el cociente del volumen del sistema lleno $V$ y el flujo volumétrico Q que atraviesa el medio, como se expresa en la ecuación 2:

$$
\bar{t}_{\text {teór }}=\mathrm{V} / \mathrm{Q}
$$

V/Q representa el llamado tiempo medio de permanencia en el caso de sistemas ideales. Comparando los resultados de las ecuaciones (1) y (2), es posible calcular los volúmenes activos de la unidad y, por diferencia de 100, el porcentaje de volúmenes estancados.

Por otro lado, la evaluación de las curvas de respuesta obtenidas puede establecer la presencia de bypasses como resultado. Esto es evidente cuando las curvas muestran dos picos. El primero de ellos refleja el fenómeno de canalización del trazador rápidamente de la entrada a la salida. El segundo representaría la fracción principal del fluido en el sistema. La relación entre las áreas bajo los dos picos nos permite cuantificar el grado o porcentaje de canalización. En general, se considera un bypass cuando una señal rápida evaluada es menos de 10-15\% del tiempo de residencia medio dado por la ecuación 1.

Esa primera ecuación rendiría los mismos resultados que la ecuación 2 si el volumen total $(V)$ de la unidad evaluada participara equitativamente en el proceso. Si esto no es completamente así, se obtiene un valor exacto para $\bar{t}$ a partir de la ecuación 1, mientras la ecuación 2 a veces lo sobreestima.

Normalmente, $\bar{t}_{\text {exp }}$ es considerado el tiempo de residencia real, ya que considera todos los fenómenos que ocurren y es encontrado experimentalmente con gran precisión con ayuda de un radiotrazador, mientras que $\bar{t}_{\text {teér }}$ debe recalcularse considerando solo el volumen activo y, en este caso, $\bar{t}_{\exp }>\bar{t}_{\text {teór }}$. Si esto no es así, existen tres posibles razones: (a) error en el dato de flujo volumétrico o caudal, (b) error en la medición de volumen total y (c) el trazador, o parte de él, se absorbe en las paredes y retorna después al sistema.

Por otro lado, la desviación del tiempo de tránsito respecto del funcionamiento ideal mencionado se estima con la siguiente ecuación:

$$
\% d=\left(1-\frac{t_{\exp }}{t_{\text {teor }}}\right) 100
$$

$\bar{t}_{\text {exp }}$ es el tiempo medio de residencia obtenido experimentalmente a partir de la curva de distribución del tiempo de residencia y $\bar{t}_{\text {teór }}$ es el tiempo medio de residencia geométrico obtenido con la ecuación 2.

\section{PROCEDIMIENTO EXPERIMENTAL}

\subsection{Instalación y prueba del sistema de inyección del radiotrazador}

Previo a la evaluación del aeroenfriador, el sistema de inyección fue instalado y probado con el personal de mantenimiento de Pluspetrol, para garantizar su funcionamiento durante la inyección del radioisótopo.

El punto de inyección se muestra en la figura 5, mientras que el sistema de inyección preparado se muestra en la figura 6 . 
INDUSTRIAL

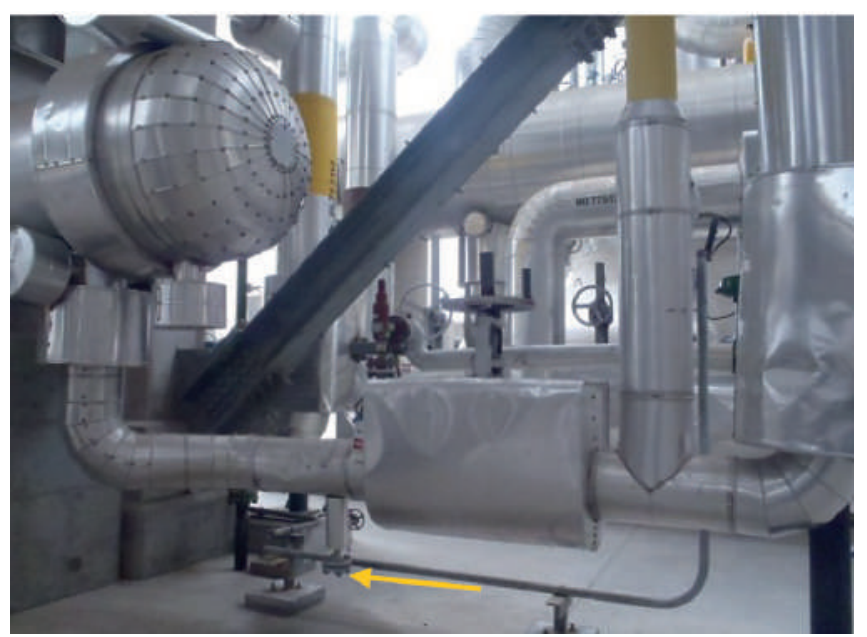

Figura 5. Zona de inyección de radiotrazador antes de su ingreso al aeroenfriador. Elaboración propia.

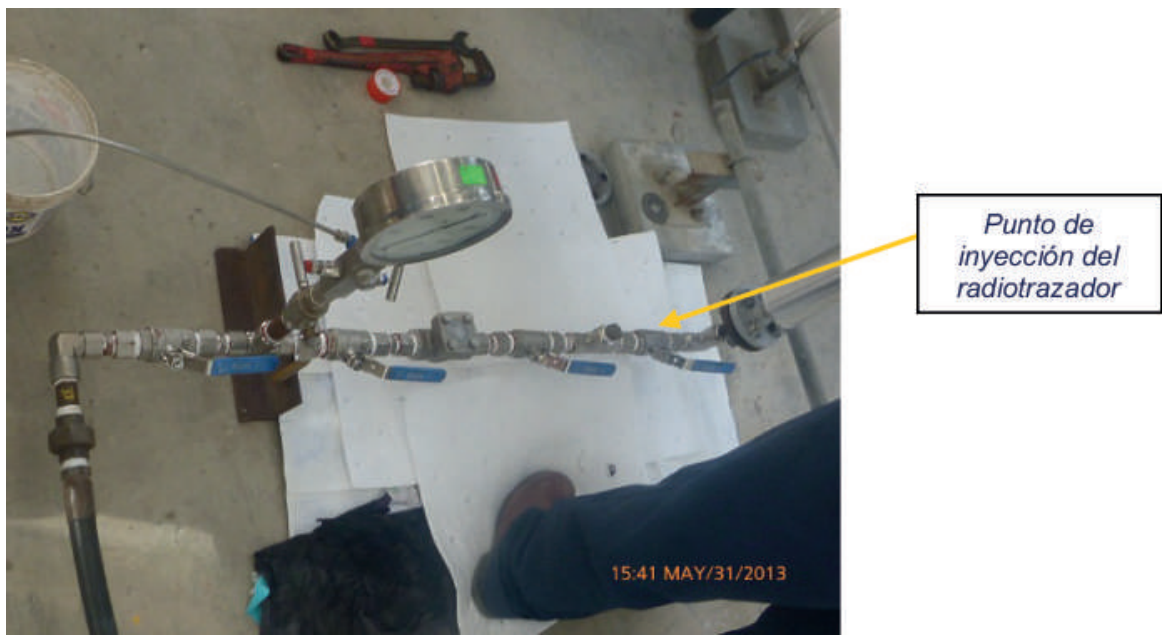

Figura 6. Sistema utilizado para la inyección del radiotrazador y punto de inyección. Elaboración propia.

El sistema de inyección se complementó con una bomba de diafragma de inyección y un cilindro con diésel, todos montados sobre el piso. La tubería, las válvulas y los demás accesorios asociados para la inyección, fueron de acero al carbono ANSI 300. Se utilizó, además, una manguera de succión del diésel contenido en el cilindro, apropiada para estos casos.

\subsubsection{Prueba del sistema de inyección de radiotrazador}

- Instalación del sistema de inyección sobre la línea de ingreso del fluido

- Apertura de la válvula de recirculación, cierre de la válvula de descarga y alineación del manómetro

- Inicio de funcionamiento de la bomba en recirculación abriendo lentamente la válvula de suministro de aire

- Regulación del flujo de recirculación regulando la presión de aire mediante la válvula de suministro 


\subsubsection{Inyección del radiotrazador}

- Instalación de la descarga de la bomba en el punto de inyección del diésel y retiro del tapón de la T ubicada en la descarga de la bomba

- Inyección de 2,5 ml. de radiotrazador por la parte superior de la T mencionada y colocación del tapón respectivo

- Verificación del ajuste del tapón de la T

- Apertura de la válvula de recirculación, cierre de la válvula de descarga y alineación del manómetro respectivo

- Inicio del funcionamiento de la bomba, abriendo lentamente la válvula de suministro de aire, regulando la presión de aire mediante la válvula de suministro

- Regulación de la presión de descarga de la bomba 100 psig (por encima de la presión de la corriente de proceso: 70 psig), para asegurar la inyección del radiotrazador

- Apertura de la válvula de inyección y de ingreso al sistema

- Simultáneamente se abre la válvula de descarga de la bomba y se cierra la de recirculación

- Se deja funcionando la bomba aproximadamente durante 30 segundos para permitir la inyección del trazador y lavado del mismo

- Parada de la bomba al detectar la inyección completa del radiotrazador en la corriente de ingreso al sistema

- Monitoreo del punto de inyección y accesorios (válvulas y check) y desmontaje del sistema de inyección (almacenando la válvula, "T" y check)

A continuación, se detallan particularidades de la técnica utilizada y los arreglos experimentales para la inyección del radiotrazador, efectuada para la determinación de eventuales obstrucciones en los tubos del aeroenfriador evaluado.

\subsection{Evaluación de la corriente del diésel que circula por los tubos a través del aeroenfriador}

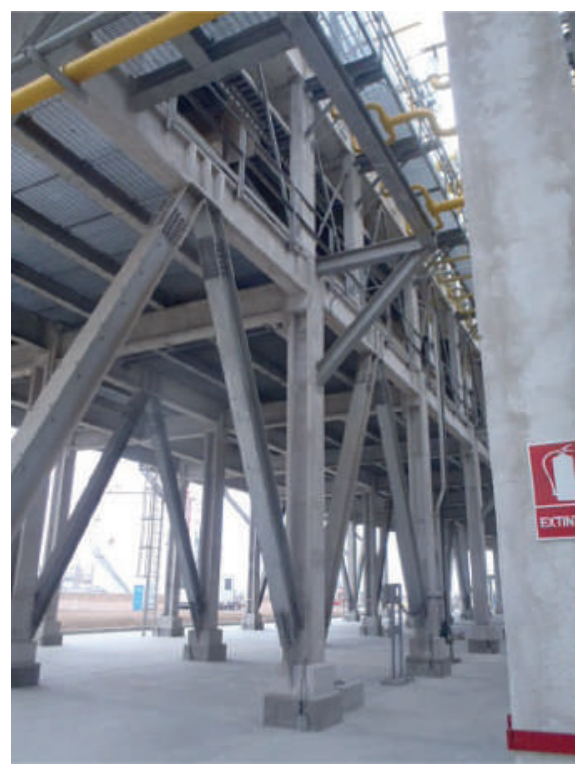

El esquema experimental fue montado considerando la inyección en la línea de entrada al aeroenfriador evaluado, complementándose con la colocación de detectores de radiación gamma ubicados según se ha graficado en las figuras 5 y 6 .

La inyección del radiotrazador se realizó en la corriente de Diésel que ingresa al sistema para recorrer los tubos del aeroenfriador.

La detección del radiotrazador se realizó en puntos claves del sistema, de acuerdo al detalle de las figuras 1, 2 y 3 , si se toma como referencia el plano proporcionado por la empresa.

Figura 7. Vista inferior de

la estructura de soporte del

aeroenfriador evaluado.

Elaboración propia. 
En las figuras 7, 8 y 9 se observan las diferentes partes del sistema evaluado, donde se ubicaron los diferentes sistemas de detección y el sistema de adquisición de datos utilizado para el registro y posterior procesamiento de la información.

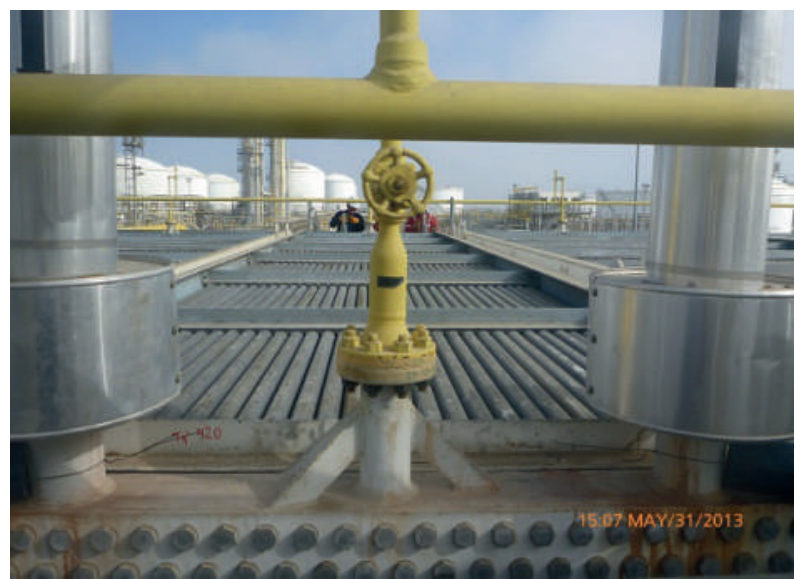

Figura 8. Vista frontal del aeroenfriador evaluado, donde se observan las dos entradas al distribuidor ubicado en el nivel superior del sistema. Elaboración propia.

Los detectores fueron dispuestos según el detalle mostrado en las figuras. 9, 10, 11 y 12.

Detectores 1 y 2 (D1 y D2): al ingreso de diésel en los dos ductos de entrada al aeroenfriador.

Detector 3 (D3): sobre el final de los tubos $\mathrm{N}^{\circ} 3$ y $\mathrm{N}^{\circ} 4$, al extremo opuesto del ingreso del aeroenfriador, en el distribuidor de pase del fluido del primer paso o nivel superior al segundo paso de la instalación. Este detector registrará, además, el final del recorrido por el tercer paso del aeroenfriador.

Detector 4 (D4): sobre el final entre los tubos $\mathrm{N}^{\circ} 10$ y N $\mathrm{N}^{\circ} 11$ en el distribuidor de pase del fluido del primer paso o nivel superior al segundo paso de la instalación. Este detector registrará, también, el final del recorrido por el tercer paso del aeroenfriador.

Detector 5 (D5): sobre el final del tubo $\mathrm{N}^{\circ} 20$, en el distribuidor de pase del fluido del primer paso o nivel superior al segundo paso al segundo paso de la instalación. Este detector registrará, incluso, el final del recorrido por el tercer paso del aeroenfriador.

Detector 6 (D6): sobre el final de los tubos $\mathrm{N}^{\circ} 23$ y N 24 , en el distribuidor de pase del fluido del primer paso o nivel superior al segundo paso al segundo paso de la instalación. Este detector registrará, además, el final del recorrido por el tercer paso del aeroenfriador.

Detector 7 (D7): Sobre el final de los tubos $\mathrm{N}^{\circ} 30$ y N³1, en el distribuidor de pase del fluido del nivel del primer paso o nivel superior al segundo paso al segundo paso de la instalación. Este detector registrará, también, el final del recorrido por el tercer paso del aeroenfriador.

Detector 8 y 9 (D8 y D9): A la salida del sistema aeroenfriador, luego del retorno del diésel por el cuarto paso (nivel inferior). 


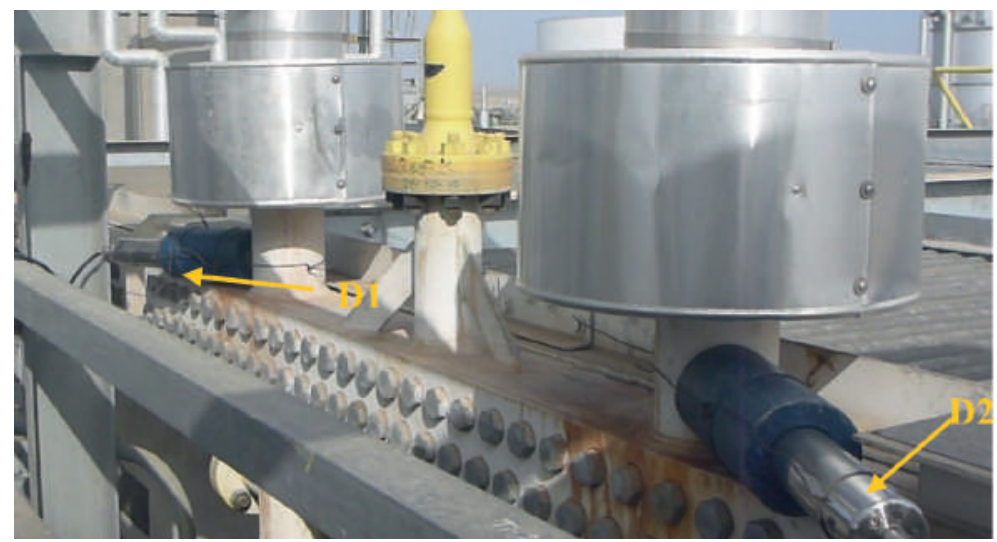

Figura 9. Vista panorámica del arreglo experimental de la instalación de los detectores D1 (izquierda) y D2 (derecha) al ingreso a los ductos de entrada al distribuidor del aeroenfriador evaluado.

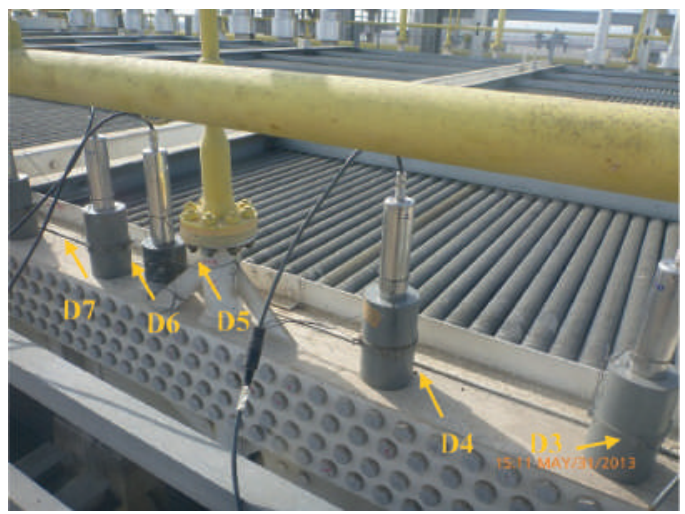

Figura 10. Arreglo experimental de detectores (de derecha a izquierda: D3, D4, D5, D6 y D7), a la salida del primer paso de los tubos por el nivel superior y retorno por el segundo paso de tubos del aeroenfriador. Estos detectores registrarán, además, el final del recorrido por el tercer paso del sistema. Elaboración propia.

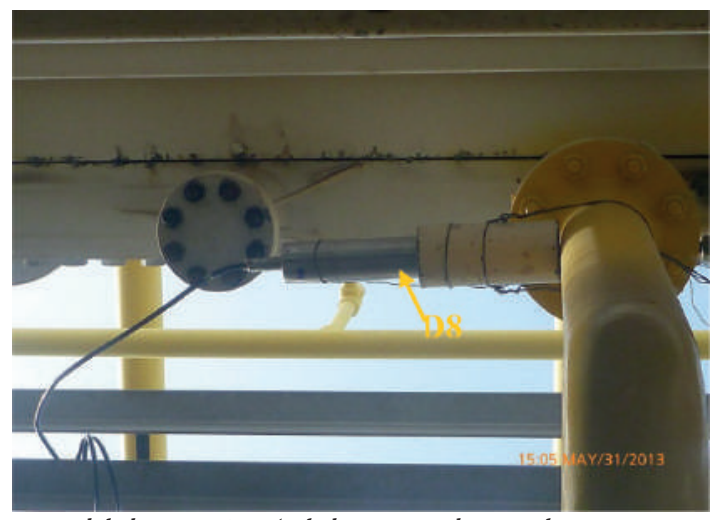

Figura 11. Ubicación del detector D8 (salida izquierda por el cuarto paso o nivel inferior del aeroenfriador). Elaboración propia. 


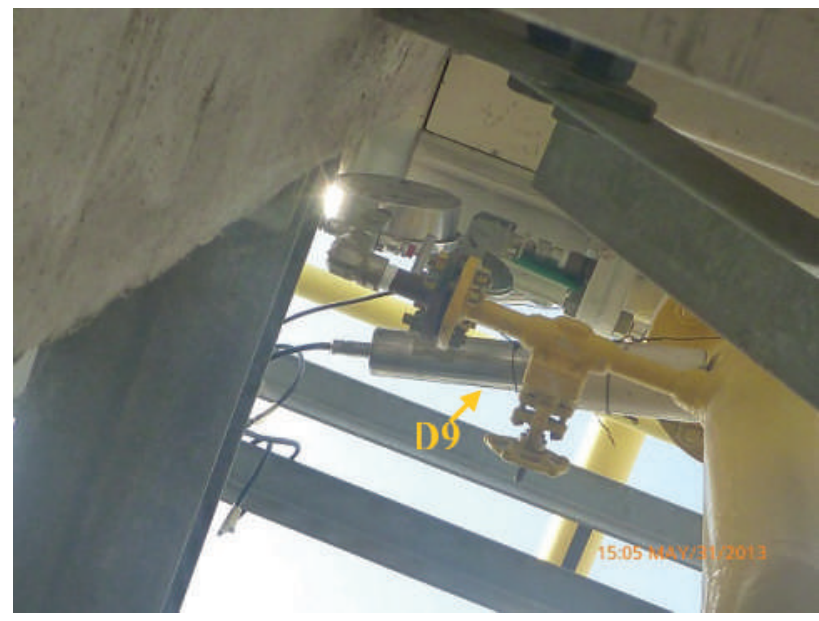

Figura 12. Ubicación del detector D9 (salida derecha por el cuarto paso o nivel inferior del aeroenfriador). Elaboración propia.

\section{TABLA DE DATOS Y RESULTADOS OBTENIDOS}

A continuación, se presentan los resultados obtenidos en los puntos de inyección y detección del radiotrazador en el intercambiador de calor evaluado.

\section{Datos:}

- Caudal de trabajo (Q):

110 GPM, el 31-05-2013 a las 4:40 pm. (*)

- Número de tubos:

$\left(0.00693 \mathrm{~m}^{3} / \mathrm{s}\right)$

- Longitud de tubo/paso:

134

- Número de pasos:

$9,14 \mathrm{~m}$.

- Diámetro de tubo:

4

- Área nominal de la sección de tubos:

1 “ OD

$0.0003326 \mathrm{~m}^{2}$

- T. de tránsito total teórico estimado ( $\left.t_{\text {teér }}\right): \quad$ aprox. 58,8 s. (De acuerdo con la ecuación 2) (**)

- Intervalo de medición (conteo de radiación): Cada 0,5 s.

(*) Caudal proporcionado en el día y hora señalados

(**) No se considera el volumen de los distribuidores

\subsection{Resultados obtenidos desde la inyección del radiotrazador hasta su detección a la entrada del aeroenfriador}

Las gráficas 1 y 2 muestran las curvas de respuesta en la zona de entrada del aeroenfriador evaluado, registradas con el posicionamiento indicado de los detectores D1 y D2, según el esquema experimental mostrado en las figuras 1 y 2 . Cabe mencionar que, a pesar de que el punto de inyección se encuentra algo distante de las entradas al aeroenfriador, se observa poca dispersión del radiotrazador en la entrada al sistema evaluado, lo cual favorece notablemente la precisión de los resultados a obtener. 


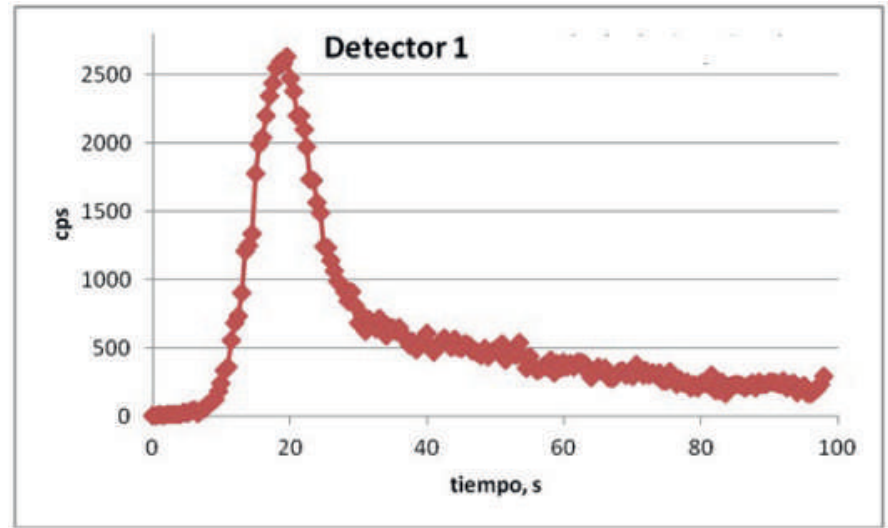

Gráfica 1. Curva de ingreso del radiotrazador al aeroenfriador por la posición del detector D1. Elaboración propia.

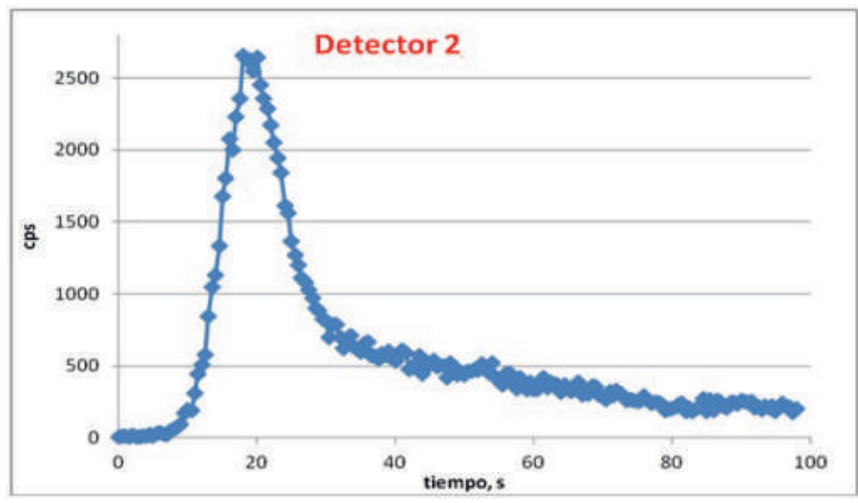

Gráfica 2. Curva de ingreso del radiotrazador al aeroenfriador por la posición del detector D2. Elaboración propia.

\subsection{Resultados obtenidos desde la entrada del radiotrazador al aeroenfriador evaluado hasta su detección a la salida por el terminal en el extremo opuesto}

Las gráficas 3, 4, 5, 6 y 7 muestran las curvas de respuesta en la zona del terminal en el extremo opuesto a la entrada del aeroenfriador evaluado, registradas con el posicionamiento indicado de los detectores D3, D4, D5, D6 y D7, según el esquema experimental mostrado en las figuras 1 y 3. Se aprecian las diferentes fracciones de radiotrazador que son registradas varias veces por cada uno de estos detectores, las mismas que serán afinadas con la utilización del software Latin 36, utilizado para estos fines. 
INDUSTRIAL

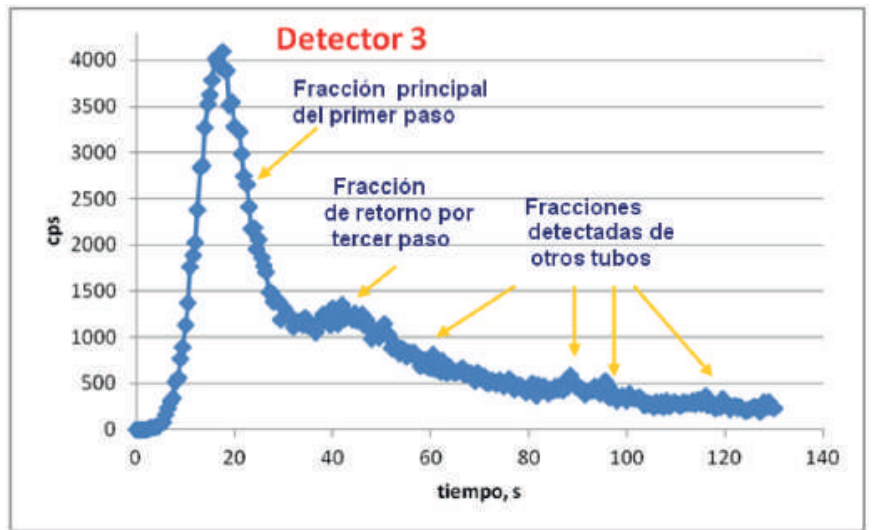

Gráfica 3. Curva de paso del radiotrazador por la posición del detector D3 (entre tubos 3 y 4). Elaboración propia.

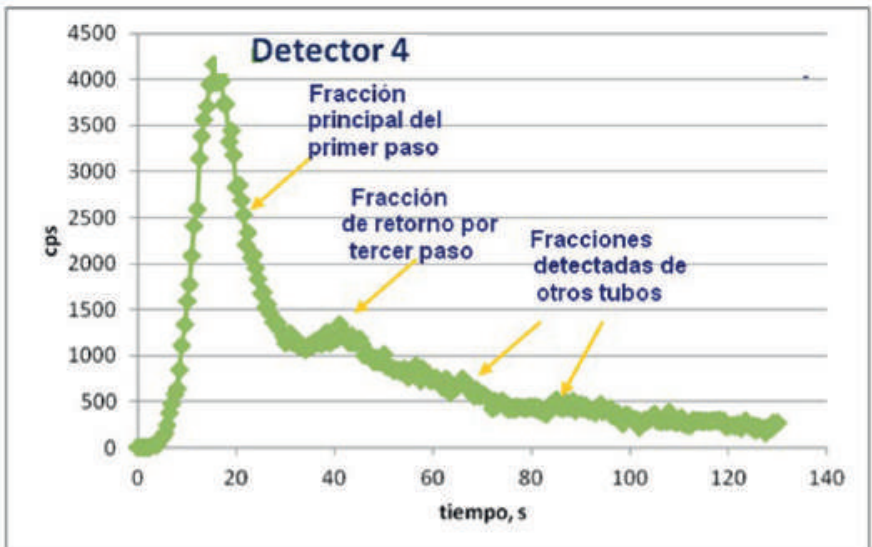

Gráfica 4. Curva de paso del radiotrazador por la posición del detector D4 (entre tubos 10 y 11). Elaboración propia.

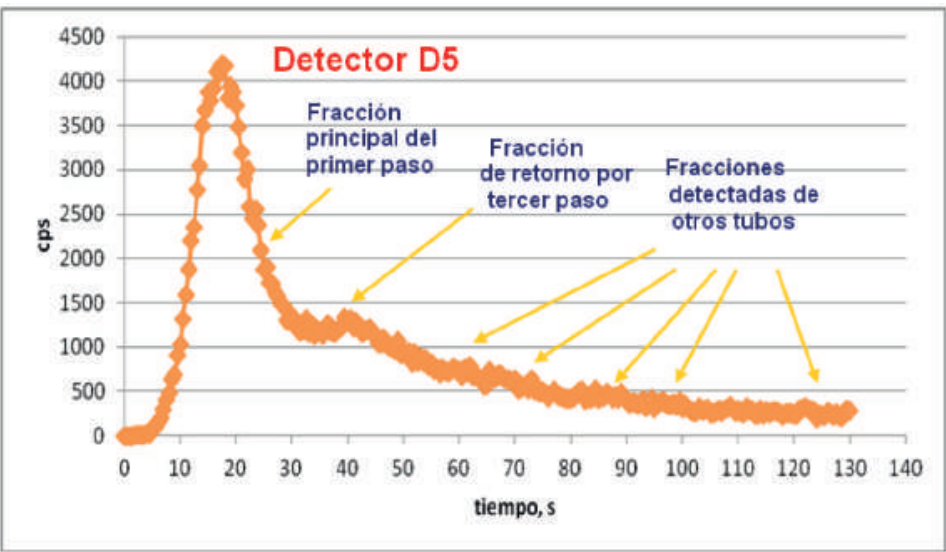

Gráfica 5. Curva de paso del radiotrazador por la posición del detector D5 (sobre el final tubo 20). Elaboración propia. 


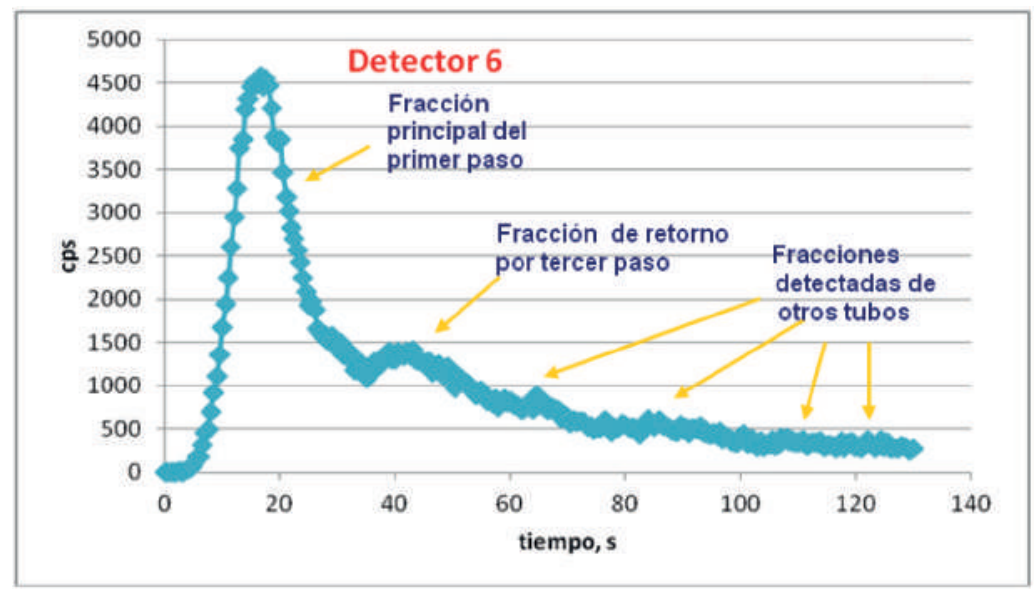

Gráfica 6. Curva de paso del radiotrazador por la posición del detector D6 (entre final tubos 23 y 24). Elaboración propia.

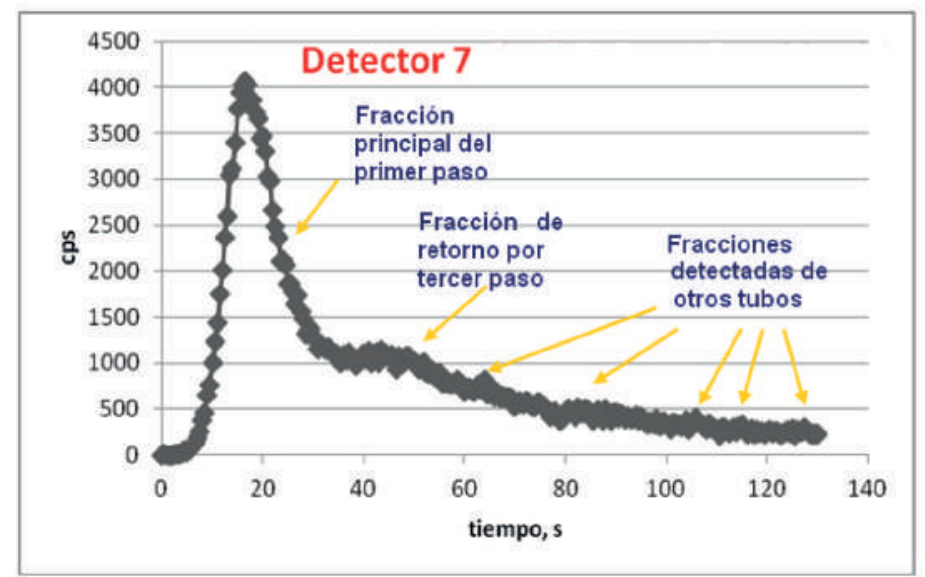

Gráfica 7. Curva de paso del radiotrazador por la posición del detector D7 (entre final de tubos 30 y 31). Elaboración propia.

Los primeros resultados de la evaluación de las curvas de respuesta mencionadas se muestran en las tablas 1 y 2 , donde se indican, respectivamente, los tiempos de tránsito en el paso uno y desde la entrada al segundo paso hasta el terminal del tercer paso (entrada al cuarto paso sin considerar la influencia del terminal). 


\begin{tabular}{|c|c|c|c|}
\hline Paso 1 (descripción) & $\begin{array}{c}\text { Tiempo de tránsito neto } \\
\text { desde la entrada hasta la } \\
\text { posición de los detectores } \\
\text { t (s) }\end{array}$ & $\begin{array}{c}\text { Pico máximo de la } \\
\text { curva (cps) }\end{array}$ & Área bajo la curva \\
\hline $\begin{array}{c}\text { Desde la entrada hasta la } \\
\text { posición de D3 }\end{array}$ & 12,5 & 4093 & 123472 \\
\hline $\begin{array}{c}\text { Desde la entrada hasta la } \\
\text { posición de D4 }\end{array}$ & 11,0 & 4165 & 118894 \\
\hline $\begin{array}{c}\text { Desde la entrada hasta la } \\
\text { posición de D5 }\end{array}$ & 12,0 & 4185 & 125082 \\
\hline $\begin{array}{c}\text { Desde la entrada hasta la } \\
\text { posición de D6 }\end{array}$ & 11,5 & 4576 & 129190 \\
\hline $\begin{array}{c}\text { Desde la entrada hasta la } \\
\text { posición de D7 }\end{array}$ & 13,5 & 4072 & - \\
\hline $\begin{array}{c}\text { Tiempo de tránsito prome- } \\
\text { dio en el Paso 1 }\end{array}$ & $12,1 \mathrm{~s}$ & - & \\
\hline
\end{tabular}

Tabla 1. Tiempos de tránsito experimentales obtenidos desde la entrada al aeroenfriador hasta el distribuidor (nivel superior o paso 1 del diésel). Elaboración propia.

\begin{tabular}{|c|c|}
\hline Pasos 2 a 4 (descripción) & $\mathrm{t}(\mathrm{s})$ \\
\hline $\begin{array}{c}\text { Desde la entrada al segundo paso hasta la salida del tercer } \\
\text { paso (registrado por el Detector 3) }\end{array}$ & 35,5 \\
\hline $\begin{array}{c}\text { Desde la entrada al segundo paso hasta la salida del tercer } \\
\text { paso (registrado por el Detector 4) }\end{array}$ & 37,2 \\
\hline $\begin{array}{c}\text { Desde la entrada al segundo paso hasta la salida del tercer } \\
\text { paso (registrado por el Detector 5) }\end{array}$ & 35,0 \\
\hline $\begin{array}{c}\text { Desde la entrada al segundo paso hasta la salida del tercer } \\
\text { paso (registrado por el Detector 6) }\end{array}$ & 35,5 \\
\hline $\begin{array}{c}\text { Desde la entrada al segundo paso hasta la salida del tercer } \\
\text { paso (registrado por el Detector 7) }\end{array}$ & 40,6 \\
\hline Tiempo de tránsito promedio & 36,6 \\
\hline
\end{tabular}

Tabla 2. Tiempos de tránsito experimentales obtenidos entre los pasos 2 y 3 (Desde la entrada al segundo paso hasta el terminal del tercer paso, doble recorrido). Elaboración propia.

\subsection{Resultados obtenidos hasta la salida del aeroenfriador evaluado}

Los resultados obtenidos a la salida del aeroenfriador evaluado se basan en el análisis de las curvas de respuesta obtenidas a la salida del sistema enfriador, registradas por los detectores D8 y D8, según el esquema experimental descrito. Las gráficas 8 y 9 muestran las curvas de respuesta a la salida del sistema aeroenfriador. 


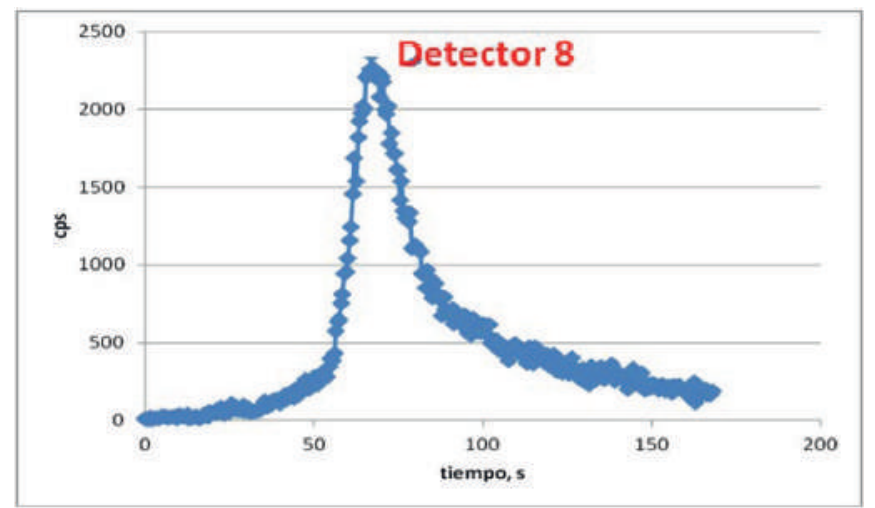

Gráfica 8. Curva de paso del radiotrazador por la posición del detector D8 (salida lado izquierdo). Elaboración propia.

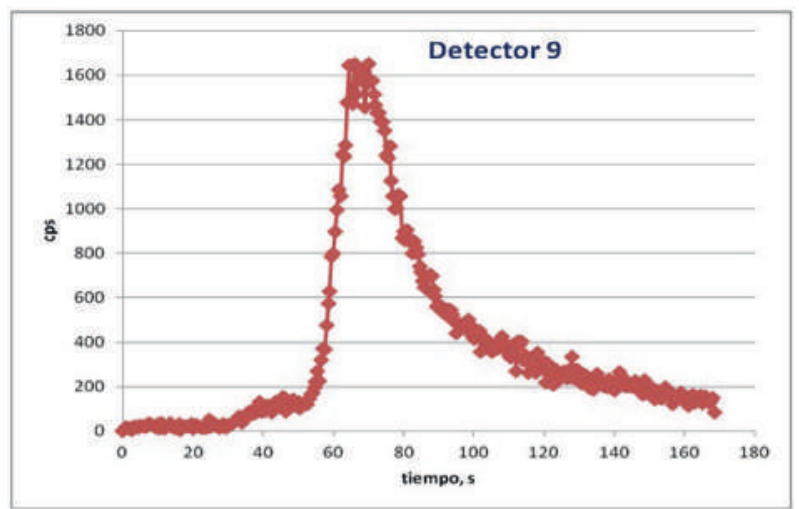

Gráfica 9. Curva de paso del radiotrazador por la posición del detector D9 (salida lado derecho). Elaboración propia.

Las tablas 3 y 4 muestran los resultados de tiempo de tránsito desde los diferentes registros de los detectores D3 a D7 hasta la salida del sistema por el nivel o paso inferior. Los registros obtenidos fueron afinados con la utilización del software Latin 36, utilizado para estos fines, y por diferencia con el tiempo total de tránsito registrado por los detectores D8 y D9, según el esquema experimental descrito.

\begin{tabular}{|l|c|}
\hline \multicolumn{1}{|c|}{$\begin{array}{c}\text { Pasos 2 a 4 } \\
\text { (descripción) }\end{array}$} & $\mathrm{t}(\mathrm{s})$ \\
\hline Desde la salida del primer paso por la posición de D3 hasta la salida del equipo & 49,0 \\
\hline Desde la salida del primer paso por la posición de D4 hasta la salida del equipo & 50,5 \\
\hline Desde la salida del primer paso por la posición de D5 hasta la salida del equipo & 51,0 \\
\hline Desde la salida del primer paso por la posición de D6 hasta la salida del equipo & 52,5 \\
\hline Desde la salida del primer paso por la posición de D7 hasta la salida del equipo & 49,5 \\
\hline Tiempo de tránsito promedio & 50,5 \\
\hline
\end{tabular}

Tabla 3. Tiempos de tránsito experimentales obtenidos desde la salida del primer paso del nivel superior hasta la salida del aeroenfriador en el nivel inferior (salida del cuarto paso). Elaboración propia. 


\begin{tabular}{|l|c|}
\hline \multicolumn{1}{|c|}{ Paso 4 (descripción) } & $\mathrm{t}(\mathrm{s})$ \\
\hline Desde la salida del tercer paso (D3) hasta la salida del Aeroenfriador & 13,5 \\
\hline Desde la salida del tercer paso (D4) hasta la salida del Aeroenfriador & 13,3 \\
\hline Desde la salida del tercer paso (D5) hasta la salida del Aeroenfriador & 14,5 \\
\hline Desde la salida del tercer paso (D6) hasta la salida del Aeroenfriador & 16,0 \\
\hline Desde la salida del tercer paso (D7) hasta la salida del Aeroenfriador & 8,9 \\
\hline Tiempo de tránsito promedio (s) & 13,5 \\
\hline
\end{tabular}

Tabla 4. Tiempos de tránsito experimentales obtenidos en el cuarto paso. Elaboración propia.

Las tablas 5 y 6 muestran los resultados consolidados de tiempos de tránsito experimentales en cada tramo evaluado y el tiempo de tránsito experimental total.

\begin{tabular}{|c|c|}
\hline Paso & $\begin{array}{c}\text { Tiempo de tránsito promedio total } \\
\text { estimado }(\mathrm{s})\end{array}$ \\
\hline 1 & 12,1 \\
\hline $2-3$ & 36,6 \\
\hline 4 & 13,5 \\
\hline Paso 1 a 4 & 62,3 \\
\hline
\end{tabular}

Tabla 5. Tiempos de tránsito experimentales en cada paso. Elaboración propia.

\begin{tabular}{|l|l|c|}
\hline $\begin{array}{c}\text { Detectores que } \\
\text { registran el } \\
\text { recorrido total } \\
\text { en el sistema }\end{array}$ & Paso 1 al paso 4 (Descripción) & $\begin{array}{c}\text { Tiempo de } \\
\text { tránsito (s) }\end{array}$ \\
\hline D1 y D8 & Primera entrada a aeroenfriador (D1) hasta primera salida (D8) & 61,5 \\
\hline D2 y D9 & Segunda entrada a aeroenfriador (D2) hasta segunda salida, (D9) & 63,0 \\
\hline $\begin{array}{c}\text { Tiempo de } \\
\text { tránsito total } \\
\text { promedio }(s)\end{array}$ & 62,3 \\
\hline
\end{tabular}

Tabla 6. Tiempo de tránsito total. Elaboración propia.

La tabla 7 muestra la desviación del tiempo de tránsito total experimental obtenido para el sistema, respecto del funcionamiento ideal.

\begin{tabular}{|c|c|c|}
\hline $\begin{array}{c}\text { Tiempo de tránsito } \\
\text { teórico, asumiendo un } \\
\text { comportamiento ideal }(\mathrm{s})(*)\end{array}$ & $\begin{array}{c}\text { Tiempo de tránsito } \\
\text { experimental promedio }(\mathrm{s})\end{array}$ & $\begin{array}{c}\text { Desviación respecto del } \\
\text { funcionamiento ideal }(* *) \text {, en \% }\end{array}$ \\
\hline 58,8 & 62,3 & 5,86 \\
\hline
\end{tabular}

Tabla 7. Desviación del tiempo con respecto al funcionamiento ideal. Elaboración propia.

(*) Estimado en base a la Ecuación 2.

(**) Estimado en base a la Ecuación 3. 
Finalmente, en la tabla 8 se muestran los resultados de los cálculos consolidados de velocidades medias y secciones efectivas medias en cada paso del sistema enfriador.

\begin{tabular}{|c|c|c|c|c|c|c|c|}
\hline Paso & $\mathrm{t}(\mathrm{s})$ & $\begin{array}{c}\text { Longitud del } \\
\text { paso }(\mathrm{m})\end{array}$ & $\begin{array}{c}\text { No de } \\
\text { tubos }\end{array}$ & $\begin{array}{c}\text { Velocidad } \\
\text { media }(\mathrm{m} / \mathrm{s})\end{array}$ & $\begin{array}{c}\text { Sección me- } \\
\text { dia teórica de } \\
\text { tubos }\left(\mathrm{m}^{2}\right)\end{array}$ & $\begin{array}{c}\text { Sección } \\
\text { media } \\
\text { efectiva de } \\
\text { tubos }\left(\mathrm{m}^{2}\right)\end{array}$ & $\begin{array}{c}\% \text { de } \\
\text { variación }\end{array}$ \\
\hline 1 & 12,1 & 9,14 & 33 & 0,755 & 0,0109 & 0,0091 & 16,5 \\
\hline 2 a $3\left(^{*}\right)$ & 36,3 & 18,28 & 67 & 0,498 & 0,0222 & 0,0278 & 25,2 \\
\hline 4 & 13,5 & 9,14 & 34 & 0,676 & 0,0113 & 0,0102 & 9,7 \\
\hline
\end{tabular}

Tabla 8. Estimación de velocidades medias de desplazamiento del diésel y áreas efectivas de los tubos en cada paso. Elaboración propia.

(*) Tiempos de tránsito promedio, considera el tiempo de tránsito en el distribuidor

\section{ANÁLISIS Y DISCUSIÓN DE RESULTADOS}

- La señal de ingreso (función de entrada) está determinada por el ingreso del diésel con el radiotrazador inyectado al aeroenfriador. Se registra el mismo comportamiento en ambos detectores (D1 y D2) ubicados a la entrada del aeroenfriador (gráficas 1 y 2), con poca dispersión.

- Los tiempos de tránsito del diésel en la zona superior en el primer paso del aeroenfriador, desde el ingreso al sistema (D1 y D2) hasta la salida del primer paso por las posiciones de los detectores D3, D4, D5, D6 y D7, ubicados en el terminal opuesto al ingreso, también con distribuidor, (primeros 9,14 metros de recorrido del diésel), son de $12,5 \mathrm{~s}, 11 \mathrm{~s}, 12 \mathrm{~s}, 11,5 \mathrm{~s}$ y 13,5 s, respectivamente, con un promedio de $12,1 \mathrm{~s}$. (véase la tabla 1). Estos resultados nos sugieren un comportamiento dinámico un tanto desigual en el primer paso, con tendencia a ser más lento en el extremo derecho del aeroenfriador evaluado.

- Los tiempos de tránsito en la zona inferior del aeroenfriador, desde la salida del paso 1 (D3, D4, D5, D6 y D7) hasta la salida del aeroenfriador (posición de los detectores D8 y D9), son de 49,0 s, 50,5 s, 51,0 s y 52,5 s, con un promedio de 50,5 segundos (véase la tabla 2), con tendencia a uniformizarse por efecto del distribuidor y el terminal, ya que, para ser tiempos de tramos ya bastante largos, las diferencias en proporción de variación no son tan considerables.

- En las curvas de respuesta de los detectores D3 hasta D7, se observa que todas tienen un ligero incremento posterior a la curva de distribución principal. Esto se debe a que los detectores (D3, D4, D5, D6 y D7) están registrando el tránsito del diésel con el radiotrazador del tercer al cuarto paso, lo cual resulta en un tiempo de tránsito aproximado, entre los pasos 2 y 3 , de $35,5 \mathrm{~s}, 37,2$ s, 35,0 s, 35,5 s y 40,6 s, respectivamente, con un promedio de 36,6 s (véanse las gráficas 3, 4, 5, 6 y 7 y la tabla 3) y un tiempo de tránsito estimado, en cada paso, de 18,3 s.

- A partir de los resultados obtenidos en el párrafo anterior, es posible calcular los tiempos de tránsito del diésel en el cuarto paso, los cuales, según la posición de los detectores D3, D4, D5, D6, D7, serían de 13,5 s, 13,3 s, 14,5 s, 16,0 s, y 8,9 s, respectivamente, con un promedio de 13,5 $s$ (véase la tabla 4). Asimismo, existe una tendencia al tránsito preferencial en el tubo del cuarto paso hacia la salida del sistema, aunque, en términos generales, por el tiempo de tránsito total, el sistema guarda bastante uniformidad hasta el momento. 
- Considerando el flujo proporcionado (110 gal/min.) al momento de su ingreso al aeroenfriador, se estima que el tiempo de tránsito teórico del diésel, desde su entrada al sistema hasta la salida de este, es de 58,8 s bajo condiciones ideales de funcionamiento. Si se considera, además, una longitud total de tuberías de 36,56 m cada una (cuatro pasos de tubería de 9,14 m cada uno, sin considerar el volumen del distribuidor), se puede estimar una desviación del tempo de tránsito teórico respecto del funcionamiento ideal del orden de 5,86 \% (tablas 6 y 7).

- La eficiencia estimada del sistema resulta ser del orden de 96,65\%, sin considerar la probable influencia del volumen de los distribuidores del inicio y terminal de pasos.

- Usando el dato de caudal del diésel proporcionado por la empresa, la geometría del sistema evaluado y los resultados del tiempo de tránsito obtenidos en los diferentes pasos de tubos, se muestran las velocidades medias de desplazamiento del diésel, así como las secciones efectivas medias en cada paso, en la tabla 8 . Se observa un desplazamiento más acelerado en los pasos 1 y 4 del sistema evaluado, donde justamente las secciones efectivas medidas son menores.

\section{CONCLUSIONES}

- El tiempo promedio de tránsito experimental del diésel en el primer paso es de 12,1 s.

- El tiempo promedio de tránsito experimental del diésel entre los pasos 2 y 4 es de 50,5 s.

- El tiempo de tránsito experimental del diésel entre los pasos 2 y 3 es aproximadamente de 36,6 s, con un promedio del orden de 18,3 s.

- El tiempo promedio de tránsito experimental del diésel en el cuarto paso es de 13,5 s.

- El tiempo de tránsito experimental total obtenido por el paso del diésel a lo largo de la sección tubular del aeroenfriador EAL 24570, en la condición de operación dada, es de 58,8 s.

- La desviación del tiempo de tránsito respecto del funcionamiento ideal del aeroenfriador, estimada en las actuales condiciones de funcionamiento, es de 5,86\%.

- Como consecuencia de los resultados mostrados en la Tabla 8, existe una tendencia a los tránsitos rápidos en los tramos 1 y 4 , donde se presentan las menores secciones efectivas de los tubos del aeroenfriador.

\section{RECOMENDACIONES}

- Realizar el mantenimiento del aeroenfriador EAL 24570 a fin de mejorar su eficiencia.

- Estudiar los fenómenos de transporte de agentes que podrían afectar la eficiencia del Aeroenfriador EAL 24570, verificando las condiciones de operación y los parámetros de control de las unidades de procedencia del diésel que ingresa al sistema evaluado en esta oportunidad.

- Durante las inspecciones futuras, debe ponerse énfasis en los pasos 1 y 4, donde se presentan las menores secciones efectivas de tubos.

- Después de hacerse el mantenimiento correctivo, sería recomendable realizar una evaluación con radiotrazadores de dicha unidad, a fin de contar con una línea de base que sirva para posteriores evaluaciones. 


\section{REFERENCIAS BIBLIOGRÁFICAS}

[1] IAEA Radiotracer Residence Time Distribution Method for Industrial and Environmental Applications - 2008.

[2] IAEA "Radiotracer Technology for Engineering Unit Operation Studies and Unit Processes Optimization", Technical Report, Krakow-Poland, 1999

[3] Leclerc J.-P.: “Traceurs and tracing methods”, Récents Progrès en Génie des procédés, 79, Vol. 15, (2001).Leclerc J.-P., Grevillot G. "Traceurs et méthodes de traçages”, Récents Progrès en Génie des procédés, 61, Vol. 12, 1998.

[1] Leclerc J.-P., Grevillot G.: "Traceurs et méthodes de traçages”, Récents Progrès en Génie des procédés, 61, Vol. 12, (1998).

[4] Sebastián C., Maghella, G., Mamani, E. "Evaluación de las unidades de tratamiento de agua, utilizando técnicas de trazadores radiactivos”. Informe Técnico IPEN, Lima -Perú, 1998.

[5] Sebastian C. y otros. "Guía para Aplicaciones Industriales de Radiotrazadores y Fuentes Selladas de Radiación”. OIEA-ARCAL XLIII, 2000. 